\title{
Characterization of acid-tolerance-associated small RNAs in clinical isolates of Streptococcus mutans: Potential biomarkers for caries prevention
}

\author{
WENHUI ZHU ${ }^{1 *}$, SHANSHAN LIU ${ }^{1 *}$, PEILIN ZHUANG ${ }^{2}$, JIA LIU ${ }^{1}$, YAN WANG ${ }^{1}$ and HUANCAI LIN ${ }^{1}$ \\ ${ }^{1}$ Guanghua School of Stomatology, Hospital of Stomatology, Sun Yat-Sen University, \\ Guangdong Provincial Key Laboratory of Stomatology, Guangzhou, Guangdong 510055; ${ }^{2}$ Department of Stomatology, \\ Sun Yat-Sen Memorial Hospital, Sun Yat-Sen University, Guangzhou, Guangdong 510120, P.R. China
}

Received February 6, 2017; Accepted August 18, 2017

DOI: $10.3892 / \mathrm{mmr} .2017 .7751$

\begin{abstract}
Streptococcus mutans is a cariogenic bacterium that contributes to dental caries due to its ability to produce lactic acid, which acidifies the local environment. The potential of $S$. mutans to respond to environmental stress and tolerate low $\mathrm{pH}$ is essential for its survival and predominance in caries lesions. Small noncoding RNAs (sRNAs) have been reported to be involved in bacterial stress and virulence. Few studies have investigated the sRNAs of $S$. mutans and the function of these sRNAs remains to be elucidated. In the present study, the association between sRNA133474 and acid tolerance, including potential underlying mechanisms, were investigated within clinical strains of $S$. mutans. From pediatric dental plaques, 20 strains of $S$. mutans were isolated. An acid killing assay was performed to analyze acid tolerance of $S$. mutans. Expression patterns of sRNA133474 were investigated during various growth phases under various acidic conditions via reverse transcription-quantitative polymerase chain reaction. RNA predator and Kyoto Encyclopedia of Genes and Genomes analyses were performed to predict target mRNAs of sRNA133474 and to examine the involvement of putative pathways of target mRNAs, respectively. The results of the present study demonstrated that sRNA133474 activity was growth phase-dependent, and two distinct expression patterns were identified in 10 clinical strains. At pH 5.5 and 7.5 the expression levels of sRNA133474 were significantly different, and high-acid tolerant strains exhibited reduced expression levels of sRNA133474 compared with
\end{abstract}

Correspondence to: Professor Huancai Lin, Guanghua School of Stomatology, Hospital of Stomatology, Sun Yat-Sen University, Guangdong Provincial Key Laboratory of Stomatology, 56 Lingyuanxi Road, Guangzhou, Guangdong 510055, P.R. China E-mail: linhcgz@126.com

*Contributed equally

Key words: small noncoding RNA, clinical strains, streptoccous mutans, acid tolerance, dental caries low-acid tolerant strains. A correlation between sRNA133474 expression levels and acid tolerance was observed in 20 clinical isolates of $S$. mutans $(\mathrm{r}=-0.6298, \mathrm{P}<0.01)$. Finally, five target mRNAs (liaS, liaR, comE, $\operatorname{cov} R$ and ciaR) involved in the two-component system (TCS) were selected for further evaluation; the expression levels of three target mRNAs (liaR, ciaR and $\operatorname{cov} R$ ) were negatively correlated with sRNA133474 expression levels. In conclusion, the results of the present study suggested that $S$. mutans may utilize sRNA133474 to orchestrate TCSs for optimal adaption to acidic $\mathrm{pH}$ in clinical strains.

\section{Introduction}

As the most common bacterial-associated infectious oral disease, early childhood caries remains a serious public health challenge worldwide $(1,2)$. Despite advances in dentistry, caries remains problematic, particularly for people in disadvantaged socioeconomic groups $(3,4)$. The Third National Oral Health Survey in China reported that $66 \%$ of 5 -year-old children had a history of caries, with an average of 3.5 decayed, missing or filled teeth (5). Therefore, it is imperative to address dental caries in early childhood.

Streptococcus mutans is principally the causative organism of dental caries (6). The cariogenic mechanism undertaken by $S$. mutans involves growth and glycolysis at $\mathrm{pH}<5.0$, and demineralization of the tooth surface (7-9). Therefore, the ability of $S$. mutans to tolerate low $\mathrm{pH}$ is crucial for its virulence and pathogenesis in dental caries (10). To mitigate acid stressors in the oral cavity, $S$. mutans has evolved numerous stress response pathways $(8,11)$, including two-component systems (TCSs), which are most commonly used as transcription regulators within bacteria. TCSs have been reported to permit numerous environmental stimuli including alterations in $\mathrm{pH}$ (12-14). The involvement of numerous TCSs in acid adaption has been observed in S. mutans (15). TCS-3 (ScnRK-like) is associated with cell survival at acidic pH; TCS-2 (CiaRH) is involved in environmental stress tolerance; TCS-9 affects the response to acidic conditions (12); TCS-1 (vicK) regulates intracellular $\mathrm{pH}$ homeostasis in S. mutans (16); and TCS (LiaSR) modulates acid tolerance within $S$. mutans (17). However, the underlying mechanisms of acid tolerance within $S$. mutans remain unclear. 
Over the past decade, studies have demonstrated that small non-coding RNAs (sRNAs) can be employed by bacteria to modulate gene expression, control environmental stress responses, and contribute to metabolic and virulence pathways $(18,19)$. Some TCS-associated sRNAs have been identified in various bacterial species. For example, five similar sRNAs were reported to control competence development by targeting the CiaRH TCS within S. pneumoniae (20). Similarly, a negative feedback circuit was reported to exist between EnvZOmpR TCS and the OmrA/B sRNAs (21). However, the physiological function of sRNAs in acid tolerance of $S$. mutans and whether sRNAs regulate acid responses of $S$. mutans through TCS has yet to be investigated.

Various isolates of $S$. mutans possess an assortment of virulence-associated traits; it is therefore necessary to investigate the functions of sRNA within clinical isolates of S. mutans. Strains of $S$. mutans isolated from children with caries are genetically distinct from those found in cavity-free children. Within a patient diagnosed with severe early childhood caries, 49 unique gene segments were identified from a strain of $S$. mutans (22). Our previous study revealed that children with high severity caries were associated with $S$. mutans containing thymidine at locus 168 of the $s r t A$ gene (23). A previous study using distinct strains of $S$. mutans suggested that expression patterns of virulence- and regulatory-associated genes were heterogeneous and strain-specific (24). Studies have previously used laboratory strains of S. mutans to investigate sRNAs; however, clinical strains are rarely employed.

In the present study, the association between sRNA133474 and acid tolerance were investigated in clinical strains of $S$. mutans, to explore the underlying mechanisms of acid tolerance in S. mutans. It was demonstrated that sRNA133474 was more highly expressed at $\mathrm{pH} 7.5$ compared with $\mathrm{pH} 5.5$ in UA159 and in clinical isolates. The expression levels of sRNA133474 were measured in isolates at different growth phases under different $\mathrm{pH}$ conditions. The pathways likely to involve sRNA133474 were analyzed via Kyoto Encyclopedia of Genes and Genomes (KEGG) pathway analysis. To the best of our knowledge, the present study is the first to describe an sRNA associated with acid tolerance in clinical isolates of S. mutans.

\section{Materials and methods}

Isolation of S. mutans strains. The protocol of the present study was approved by the Ethics Committee of Guanghua School of Stomatology, Sun Yat-sen University [Guangzhou, China; ERC-(2015)-8]. Clinical strains of S. mutans were isolated from 5-year old children who participated in an epidemiological survey in Guangzhou according to the protocol described by Yu et al (23). Briefly, plaque samples from 20 children were vortexed and sonicated at maximum amplitude using a sonic oscillator (UR-200; Tomy Seiko Co., Ltd., Tokyo, Japan) in an ice bath for $30 \mathrm{sec}$, followed by serial $10^{-3}$ dilutions in PBS. Subsequently, $50 \mu 1$ diluent was plated onto Mitis-Salivarius-Bacitracin agar supplemented with $20 \%$ sucrose and $0.2 \mathrm{U} / \mathrm{ml}$ bacitracin, and incubated anaerobically $\left(85 \% \mathrm{~N}_{2}, 5 \% \mathrm{CO}_{2}\right.$ and $\left.10 \% \mathrm{H}_{2}\right)$ at $37^{\circ} \mathrm{C}$ for 2 days. One clone from each sample was randomly selected based on its ability to ferment mannitol, sorbitol, raffinose, melibiose and aesculin, and hydrolyze arginine. Clinical isolates were preserved in $25 \%$ glycerol at $-80^{\circ} \mathrm{C}$ prior to use.

Bacterial strains and culture conditions. S. mutans UA159 (ATCC700610; American Type Culture Collection, Manassas, VA, USA) and clinical isolates were cultured in brain heart infusion (BHI) or tryptone, 3\%; glucose, $20 \mathrm{mM}$; yeast extract, $0.3 \%$ (TYG) medium. To investigate growth, strains were incubated in an atmosphere containing $5 \% \mathrm{CO}_{2}$. S. mutans UA159 and clinical isolates were cultured overnight in BHI broth. The cultures were then diluted 1:50 in fresh BHI broth and grown to the indicated phase [optical density at $600 \mathrm{~nm}\left(\mathrm{OD}_{600}=0.2\right.$, 0.4 and 0.7$)$ ], after which they were collected by centrifugation $\left(7,585 \mathrm{x} \mathrm{g}, 4^{\circ} \mathrm{C}, 15 \mathrm{~min}\right)$ for RNA isolation. For acid shock assays, $\mathrm{HCl}$ or $\mathrm{NaOH}$ were added to TYG medium to adjust final $\mathrm{pH}$ to 5.5 or 7.5; UA159 and clinical isolates of $S$. mutans were cultured overnight in TYG medium and diluted 1:100 with fresh TYG medium until a mid-logarithmic phase $\left(\mathrm{OD}_{600}=0.4\right)$ was achieved. Cells were subsequently divided into two aliquots, pelleted by centrifugation $\left(7,585 \mathrm{x} \mathrm{g}, 4^{\circ} \mathrm{C}, 15 \mathrm{~min}\right)$ and resuspended in TYG medium with buffer solution to maintain the $\mathrm{pH}$ at 5.5 or 7.5 for $1.5,2.5$ and $3.5 \mathrm{~h}$ for RNA isolation.

RNA isolation. Cells were collected and centrifuged $(7,585 \mathrm{x} \mathrm{g})$ at $4^{\circ} \mathrm{C}$ for $15 \mathrm{~min}$, and resuspended in $100 \mathrm{ml}$ lysis buffer (20 mg/ml lysozyme, $60 \mathrm{mAU} / \mathrm{ml}$ proteinase $\mathrm{K}$ ) at $37^{\circ} \mathrm{C}$ for $45 \mathrm{~min}$. Total RNA extraction and purification was performed using an miRNeasy Mini kit (Qiagen, $\mathrm{GmbH}$, Hilden, Germany) according to the manufacturer's protocol. RNA samples of UA159 and clinical isolates of S. mutans were prepared in triplicate.

Reverse transcription-quantitative polymerase chain reaction (RT-qPCR). In our previous study, three libraries of sRNAs were constructed and exposed to acidic conditions (25). A total of 91 sRNAs with $\geq 1,000$ mean reads were identified and nine candidates were randomly selected (Table I). sRNAs with read counts $\geq 1,000$ were selected due to a greater interest in bacterial sRNAs with many sequencing results (26). The primer sequences for the nine selected sRNAs and five mRNAs are presented in Tables II and III, respectively. The reverse primer used for qPCR was a commercialized universal primer supplied with the Mir-X miRNA qRT-PCR SYBR kit (Takara Bio, Inc., Otsu, Japan) (27-29). Total RNA was converted into cDNA using a Mir-X miRNA First-Strand Synthesis kit (Takara Bio, Inc.) according to the manufacturer's protocol. The qPCR reaction was performed on a LightCycler 480 Real-time PCR system (Roche Diagnostics GmbH, Mannheim, Germany) with a SYBR Premix Ex Taq II kit (Takara Bio, Inc.). The reaction conditions were as follows: $95^{\circ} \mathrm{C}$ for $30 \mathrm{sec}$, followed by 40 cycles of $95^{\circ} \mathrm{C}$ for $15 \mathrm{sec}$ and $63^{\circ} \mathrm{C}$ for $30 \mathrm{sec}$. Melting curve analysis was subsequently performed from $60-95^{\circ} \mathrm{C}$. RT-qPCR was used to measure sRNA133474 expression and predicted mRNA expression at different growth phases and under different $\mathrm{pH}$ conditions. The $16 \mathrm{~S}$ rRNA gene was used as an internal control. Each assay was performed in triplicate, and fold-changes in expression were calculated using the $2^{-\Delta \Delta C q}$ method (30).

Acid killing assay. An acid killing assay was conducted to assess acid tolerance according to previously published methods (6). 
Table I. sRNAs selected for reverse transcription-quantitative polymerase chain reaction.

\begin{tabular}{|c|c|c|c|c|c|}
\hline sRNA & Start location & Length & Sequence $\left(5^{\prime}-3^{\prime}\right)$ & Strands & Mismatch \\
\hline sRNA133474 & 293191 & 33 & CAGCCCTAAGCGATGTAAGCTGTGTGCTCTATT & + & 0 \\
\hline sRNA133473 & 293190 & 34 & GCAGCCCTAAGCGATGTAAGCTGTGTGCTCTATT & + & 0 \\
\hline sRNA884831 & 1930106 & 35 & $\begin{array}{l}\text { TGGGTCGCTCTGTATCTCTG } \\
\text { CGGTGGCTGTGAGTATGAAG }\end{array}$ & + & 0 \\
\hline sRNA133477 & 293194 & 30 & $\begin{array}{l}\text { TGGATTTTCATGCCTGCTG } \\
\text { GGCTGCATTACCAGAAAGGT }\end{array}$ & + & 0 \\
\hline sRNA628417 & 1327899 & 37 & CAACACAGCTCTAAAACTGTGGCAAGTCATGTCCGAA & - & 0 \\
\hline sRNA628420 & 1327902 & 34 & CACAGCTCTAAAACTGTGGCAAGTCATGTCCGAA & - & 0 \\
\hline sRNA133484 & 293201 & 23 & CGATGTAAGCTGTGTGCTCTATT & + & 0 \\
\hline sRNA628658 & 1328100 & 34 & CACAGCTCTAAAACAGAGCACTAACTGCGCTAGC & - & 0 \\
\hline sRNA628415 & 1327897 & 39 & AАCAACACAGCTCTAAAACTGTGGCAAGTCATGTCCGAA & - & 0 \\
\hline
\end{tabular}

sRNA, small noncoding RNA.

Table II. Primers for selected sRNAs.

\begin{tabular}{lll}
\hline sRNA & \multicolumn{1}{c}{ Sequence (5'-3') } & \multicolumn{1}{c}{ Primer sequence (5'-3') } \\
\hline sRNA133474 & CAGCCCTAAGCGATGTAAGCTGTGTGCTCTATT & ATGTAAGCTGTGTGCTCTATTA \\
sRNA133473 & GCAGCCCTAAGCGATGTAAGCTGTGTGCTCTATT & GCAGCCCTAAGCGATGTAAG \\
sRNA884831 & TACAAAACGTGAATCATCGGTGCCAATACAGCATT & TCATCGGTGCCAATACAGCAT \\
sRNA133477 & CCCTAAGCGATGTAAGCTGTGTGCTCTATT & GATGTAAGCTGTGTGCTCTATT \\
sRNA628417 & CAACACAGCTCTAAAACTGTGGCAAGTCATGTCCGAA & TGTGGCAAGTCATGTCCGAAA \\
sRNA628420 & CACAGCTCTAAAACTGTGGCAAGTCATGTCCGAA & TGTGGCAAGTCATGTCCGAA \\
sRNA133484 & CGATGTAAGCTGTGTGCTCTATT & CGATGTAAGCTGTGTGCTCTA \\
sRNA628658 & CACAGCTCTAAAACAGAGCACTAACTGCGCTAGC & TAAAACAGAGCACTAACTGCG \\
sRNA628415 & AACAACACAGCTCTAAAACTGTGGCAAGTCATGTCCGAA & GTGGCAAGTCATGTCCGAAA \\
\hline
\end{tabular}

sRNA, small noncoding RNA.

Briefly, stationary-phase cells of the overnight culture were harvested and immediately subjected to acid stress by incubation in $1 \mathrm{ml} \mathrm{pH} 2.8$ glycine solution for 0,30 and $60 \mathrm{~min}$ at $37^{\circ} \mathrm{C}$. Cells were washed with $1 \mathrm{ml} \mathrm{PBS}$ at $\mathrm{pH} 7.5$ to terminate the process and were then diluted with PBS for plating. Plates were incubated at $37^{\circ} \mathrm{C}$ and colonies were counted after 2 days. Bacterial survival was assessed after exposure to lethal $\mathrm{pH}$ to measure acid tolerance of $S$. mutans isolates.

Bioinformatics analysis. RNApredator (http://rna.tbi.univie .ac.at/RNApredator) was used to predict target mRNAs of sRNA133474 (31,32). KEGG pathway analyses were performed using the Database for Annotation, Visualization and Integrated Discovery (DAVID) gene annotation tool (http://david.abcc.ncifcrf.gov/) (33). A Fisher's exact test $\mathrm{P}$-value was used to denote significance.

Statistical analysis. All experiments were performed in triplicate. Data were analyzed using IBM SPSS 19.0 for Windows (SPSS; IBM Corp., Armonk, NY, USA). Data are expressed as the mean \pm standard deviation. The Shapiro-Wilk test was first used to check whether the data were parametric or not.
Two-group comparisons were performed using Student's t-test. Multiple-group comparisons were analyzed using a one-way analysis of variance or Kruskal-Wallis, followed by Bonferroni multiple comparison tests. Correlations were analyzed with a Spearman rank correlation coefficient. $\mathrm{P}<0.05$ was considered to indicate a statistically significant difference.

\section{Results}

Expression levels of sRNA133474 in clinical strains of S. mutans and S. mutans UA159. RT-qPCR was conducted to determine the expression levels of the nine selected sRNAs in UA159 and three clinical isolates. In total, six sRNAs with low $\mathrm{Cq}(\mathrm{Cq}<35)$ and three sRNAs with high $\mathrm{Cq}(\mathrm{Cq} \geq 35$; representing low expression; data not shown) were reported. Expression levels of the six sRNAs were determined in UA159 and three clinical isolates using RT-qPCR and were subsequently measured under different $\mathrm{pH}$ conditions. At $\mathrm{pH} 7.5$ and 5.5, the differentially expressed sRNAs included sRNA133474, sRNA133473 and sRNA628658 in UA159 and clinical strains (Fig. 1A and B) among which sRNA133474 was the most significantly downregulated at $\mathrm{pH} 5.5$ compared 
Table III. Primers for mRNAs.

\begin{tabular}{ll}
\hline miRNA & \multicolumn{1}{c}{ Primer sequence (5'-3') } \\
\hline 16s-F & 5'-CCTACGGGAGGCAGCAGTAG-3' \\
16s-R & 5'-CAACAGAGCTTTACGATCCGAAA-3' \\
comE-F & 5'-AGCCCATAAGCTCTGCCTTT-3' \\
comE-R & 5'-AGCGATGGCACTGAAAAAGT-3' \\
covR-F & 5'-GCTCTTTTGCAGCAAATCAAATCGT-3' \\
covR-R & 5'-GCCAATAATCGGAAAATAAAGGCGC-3' \\
ciaR-F & 5'-CAGGTGTCGTTATCCCTTTTTCAC-3' \\
ciaR-R & 5'-GCAGAGAGTGGCGTTTATGATTTG-3' \\
liaS-F & 5'-CGAGATTTGAGTTACGGCTTG-3' \\
liaS-R & 5'-GCATCCCCTTTCATTATTGG-3' \\
liaR-F & 5'-ACCAAACGATTGCTGATGAG-3' \\
liaR-R & 5'-CCTGTGGCACTAAATGATGC-3'
\end{tabular}

miRNA, micro RNA; F, forward; R, reverse.

with at pH 7.5. Therefore, sRNA133474 was selected for subsequent experiments. sRNA133474 expression levels within 10 clinical isolates at $\mathrm{pH} 7.5$ and 5.5 were subsequently measured; regardless of strain, the expression levels of sRNA133474 were consistently elevated at $\mathrm{pH} 7.5$ compared with at $\mathrm{pH} 5.5$ (Fig. 1C) when cells were grown to a mid-logarithmic phase $\left(\mathrm{OD}_{600}=0.4\right)$.

Expression patterns of sRNA133474 in different growth phases of $S$. mutans clinical strains. Characteristics associated with sRNA133474 in clinical isolates of S. mutans were analyzed by measuring growth curves of $10 \mathrm{~S}$. mutans clinical isolates cultured in $\mathrm{pH}$ 7.5 TYG medium at different growth phases (Fig. 2A). S. mutans strains of the early-logarithmic growth phase $\left(\mathrm{OD}_{600}=0.2\right)$, mid-logarithmic growth phase $\left(\mathrm{OD}_{600}=0.4\right)$, and stationary growth phase $\left(\mathrm{OD}_{600}=0.7\right)$ were cultured; subsequently, total RNA was extracted. Among the 10 strains analyzed, two distinct expression patterns of sRNA133474 were identified: Expression pattern (i), expression levels of sRNA133474 during different growth phases were downregulated from an early-logarithmic growth phase to an early stationary growth phase (Fig. 2B); expression pattern (ii), expression levels of sRNA133474 were downregulated from an early-logarithmic growth phase to a mid-logarithmic growth phase, and were then slightly upregulated in the early stationary phase (Fig. 2C). The two expression patterns were almost equally prevalent amongst the clinical strains; they were present in six and four of the strains, respectively.

Alterations of $\mathrm{pH}$ during different growth phases. $\mathrm{pH}$ was investigated during different growth phases of the clinical strains in order to understand the importance of the different expression patterns of sRNA133474. The present study demonstrated that the $\mathrm{pH}$ of the medium decreased during specific growth phases (Fig. 3A and B). For clinical strains with sRNA133474 expression pattern (i), $\mathrm{pH}$ gradually declined during the growth phase (Fig. 3B); for clinical strains with sRNA133474 expression pattern (ii), $\mathrm{pH}$ decreased to
4.69 and then slightly increased at the stationary growth phase (Fig. 3C).

Expression levels of sRNA133474 under different acid stress conditions. To clarify the association between sRNA133474 expression and acid stress, UA159 and clinical isolates of S. mutans were cultured at $\mathrm{pH} 7.5$ and 5.5 for $1.5,2.5$ and $3.5 \mathrm{~h}$. Expression levels of sRNA133474 in UA159 and clinical isolates were determined by RT-qPCR (Fig. 4A-C). Regardless of exposure time, expression levels of sRNA133474 were increased at $\mathrm{pH} 7.5$ compared with at $\mathrm{pH} 5.5$ in clinical isolates (Fig. 4B and C); whereas lower expression levels of sRNA133474 were observed in UA159 $3.5 \mathrm{~h}$ post-treatment at pH 7.5 (Fig. 4C); however, the difference was not significant (P>0.05). However, sRNA133474 expression consistently increased at $\mathrm{pH} 7.5$ in UA159 and clinical strains after $2.5 \mathrm{~h}$ compared with in $\mathrm{pH} 5.5(\mathrm{P}<0.05)$.

Association between sRNA133474 and acid tolerance of $S$. mutans. Based on acid tolerance, 10 clinical isolates were placed into two groups. Expression levels of sRNA133474 in the low-acid tolerance group were consistently greater than in the high-acid tolerance group; the greatest fold-changes occurred at pH 5.5 at $2.5 \mathrm{~h}$ (Fig. 4A-C). The association between sRNA133474 and acid tolerance was investigated at $\mathrm{pH} 5.5$ at $2.5 \mathrm{~h}$ in 20 clinical isolates of $S$. mutans. The data of the present study revealed that sRNA133474 expression levels were lower in clinical isolates with higher acid tolerance (Fig. 5A). A correlation between sRNA133474 expression and acid tolerance was observed ( $r=-0.6298, \mathrm{P}<0.01$; Fig. 5B).

Target genes prediction and functional annotation analysis. The secondary structure of sRNA133474 was predicted using the RNAfold webserver (rna.tbi.univie. ac.at/cgi-bin/RNAWebSuite/RNAfold.cgi). It was reported that sRNA133474 possessed a stem loop structure with the $\mathrm{dG}$ value of $-5.4 \mathrm{~kJ} / \mathrm{mol}$ (Fig. 6A). The possible target mRNAs of sRNA133474 were investigated using RNApredator and 1,960 mRNAs were predicted. To determine the KEGG pathways associated with the target mRNAs of sRNA133474, the DAVID gene annotation tool (http://david.abcc.ncifcrf.gov/) was applied, the chief pathways identified included: i) Metabolic pathways; ii) synthesis of secondary metabolites; iii) antibiotic synthesis; iv) amino acid biosynthesis; v) microbial metabolism in different environments; vi) ATP-binding cassette transporters; vii) carbon metabolism; viii) purine metabolism; x) pyrimidine metabolism; xi) TCS and xii) glycolysis/starch production (Fig. 6B). The TCS pathway in $S$. mutans helps to coordinate regulatory networks and gene expression in response to acid stress (15). The TCSs CiaHR, LiaSR and CovSR are considered to contribute to acid resistance of $S$. mutans. Therefore, five putative target mRNAs: comE, $\operatorname{cov} R$, ciaR, liaS and liaR involved in TCSs were chosen for investigation $(12,14,17,34)$.

Negative correlation between sRNA133474 and target $m R N A s$. To explore the possible mechanisms underlying sRNA133474-associated acid tolerance within S. mutans, the expression levels of sRNA133474 and corresponding target mRNAs were analyzed using RT-qPCR in 20 clinical 


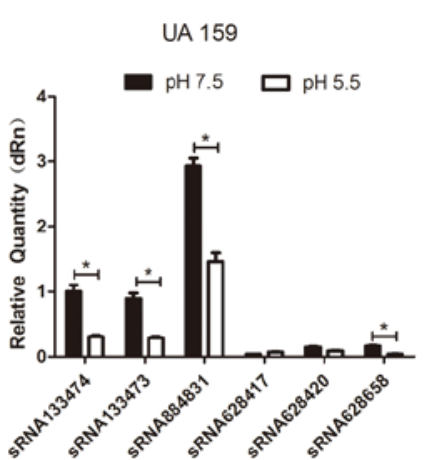

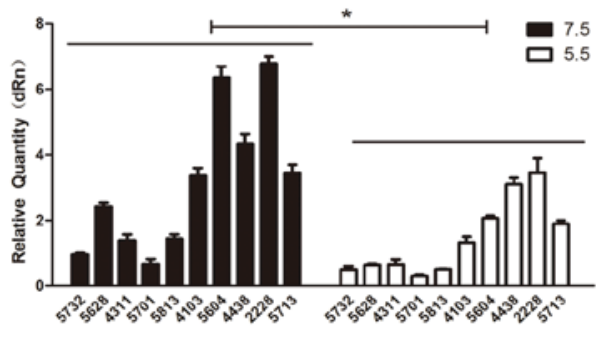

Figure 1. (A) Differential expression of six selected sRNAs at different pH environments in Streptococcus mutans UA159. Expression of sRNA133474 at pH 7.5 in UA159 is defined as 1.0. Error bars represent standard deviations of three biological replicates. (B) Differential expression of six selected sRNAs at different pH environments in three clinical strains of $S$. mutans. Mean expression of sRNA133474 at pH 7.5 in three clinical strains is defined as 1.0 . Error bars represent standard deviations of three clinical strains. (C) Expression of sRNA133474 in 10 clinical isolates at pH 7.5 and 5.5 with cells grown to the logarithmic growth phase, optical density ${ }_{600}=0.4$. Expression of sRNA133474 in strain 5732 at pH 7.5 is defined as 1.0 . Error bars represent standard deviations of three biological replicates. ${ }^{*} \mathrm{P}<0.05$ (Student's t-test). sRNA, small noncoding RNA; dRn, the magnitude of the fluorescence signal generated at each time point.
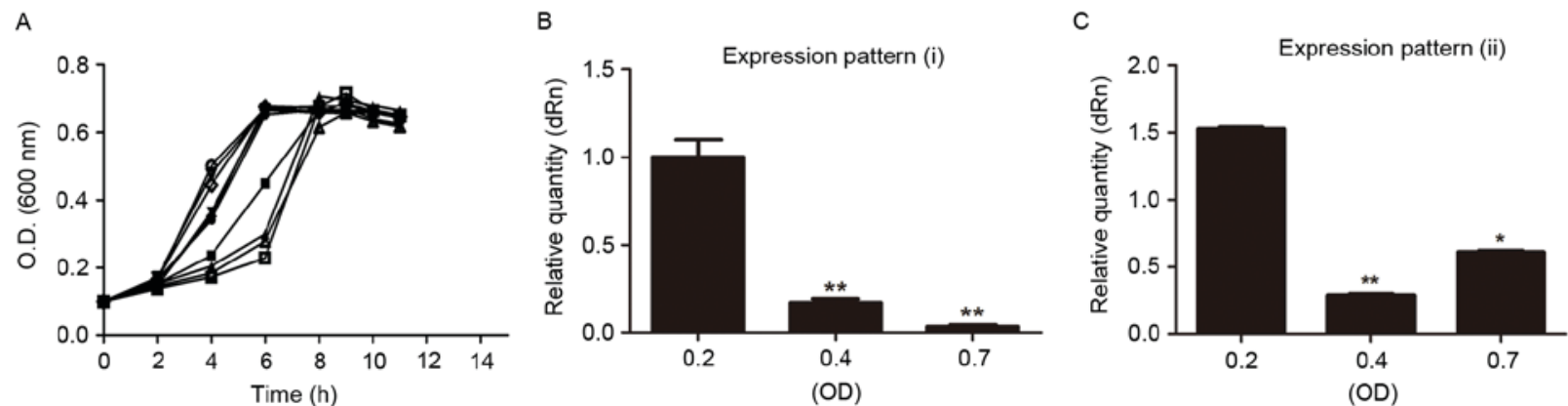

Figure 2. Characterization of sRNA133474 in clinical isolates of S. mutans. (A) Growth curves of 10 S. mutans clinical isolates cultured in pH 7.5 TYG medium. Each curve indicates one strain. (B and C) Transcription patterns of sRNA133474 during different phases of planktonic growth. Data are presented as the mean \pm standard deviation. ${ }^{*} \mathrm{P}<0.05$ and ${ }^{* *} \mathrm{P}<0.01$ compared with the relative quantity at $\mathrm{OD}_{600}=0.2$ (Kruskal-Wallis test and Bonferroni multiple comparisons test). A Kruskal-Wallis test and Bonferroni multiple comparisons tests were employed. OD, optical density; sRNA, small noncoding RNA; dRn, the magnitude of the fluorescence signal generated at each time point.

A

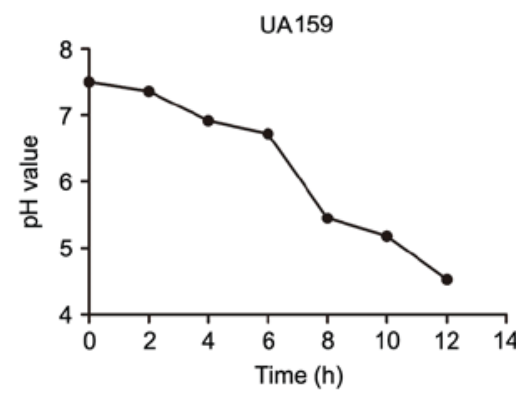

B

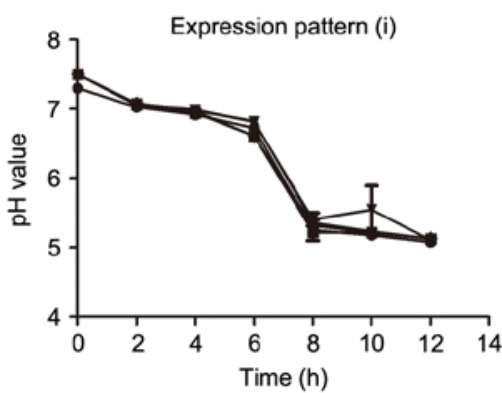

C

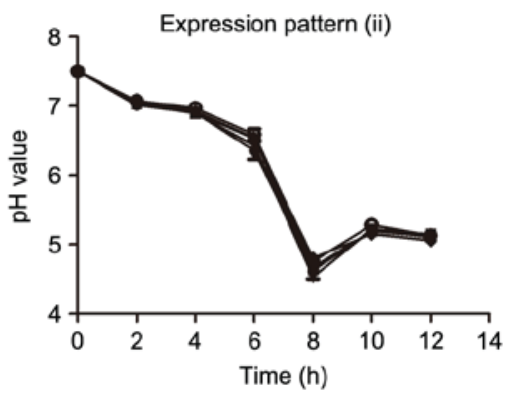

Figure 3. $\mathrm{pH}$ drop assay. Change in pH value in (A) UA159 and (B and C) in clinical strains of S. mutans with sRNA133474 expression pattern (i or ii) during different growth phases. sRNA, small noncoding RNA.

isolates with different acid resistance. Data indicated a negative correlation between sRNA133474 and mRNA liaR $(\mathrm{r}=-0.8075, \mathrm{P}<0.0001)$, ciaR $(\mathrm{r}=-0.6248, \mathrm{P}=0.0032)$ and $\operatorname{cov} R$ $(\mathrm{r}=-0.6684, \mathrm{P}=0.0013$; Fig. 7). In addition, a negative association between sRNA133474 and target mRNA during different growth phases was observed (Fig. 8). These results indicate that sRNA133474 may modulate acid tolerance of $S$. mutans, by inhibiting the expression of acid tolerance related mRNAs (liaR, $\operatorname{ciaR}, \operatorname{cov} R)$.

\section{Discussion}

As an important factor of virulence, acid tolerance permits $S$. mutans to rapidly adapt to acidic environments by modulating regulatory factors. Numerous acid tolerance-associated genes have been described; however, the molecular basis for $S$. mutans to sense and integrate $\mathrm{pH}$ signals for acid adaptation is unknown. Previously, sRNAs were identified as crucial regulators of various physiological 


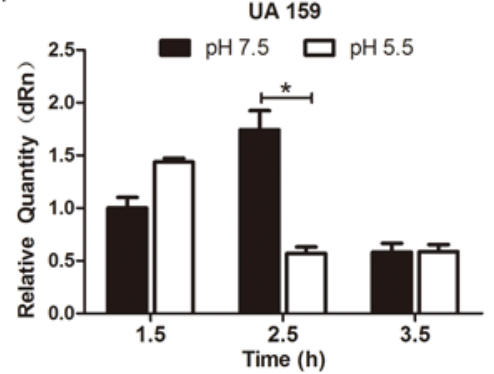

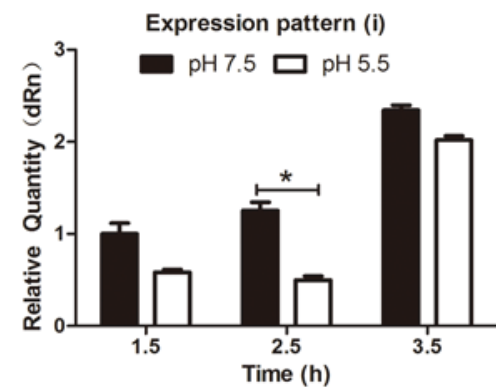

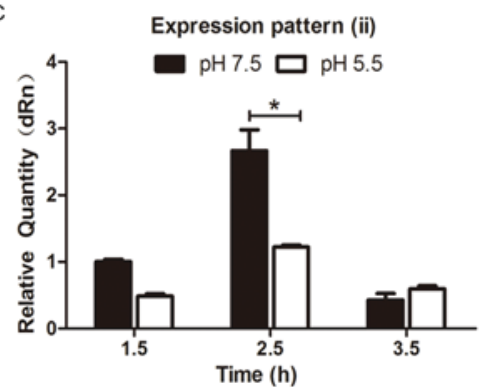

Figure 4. Reverse transcription-quantitative polymerase chain reaction analysis of sRNA133474 expression in UA159 and clinical isolates of S. mutans under different acid stress conditions. (A) Relative expression of sRNA133474 in S. mutans UA159 under pH 5.5 and 7.5 at 1.5, 2.5 and 3.5 h. (B and C) Relative expression of sRNA133474 in clinical strains under $\mathrm{pH} 5.5$ and $\mathrm{pH} 7.5$ at 1.5, 2.5 and 3.5 h. Data are presented as the mean \pm standard deviation. sRNA133474 expression at $\mathrm{pH} 7.5$ for $1.5 \mathrm{~h}$ was set as the relative value in expression patterns ( $\mathrm{i}$ and ii) respectively. Error bars show standard deviations of three replicates. ${ }^{*} \mathrm{P}<0.05$, as indicated. $\mathrm{dRn}$, the magnitude of the fluorescence signal generated at each time point.

A

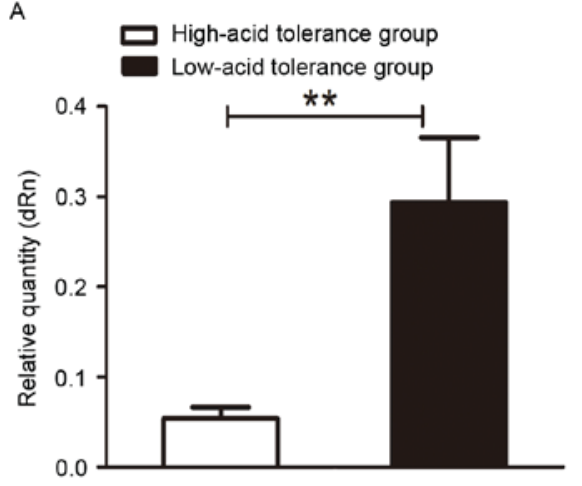

B

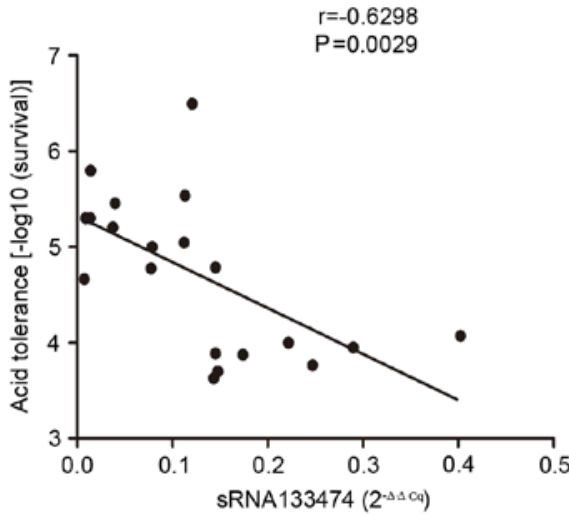

Figure 5. (A) Reverse transcription-quantitative polymerase chain reaction analysis of sRNA133474 expression in clinical strains with different acid tolerance. (B) Correlation analysis between sRNA133474 expression and acid tolerance of $S$. mutans strains $\left(\mathrm{r}=-0.6298,{ }^{* * *} \mathrm{P}<0.01\right)$. dRn, the magnitude of the fluorescence signal generated at each time point.

A

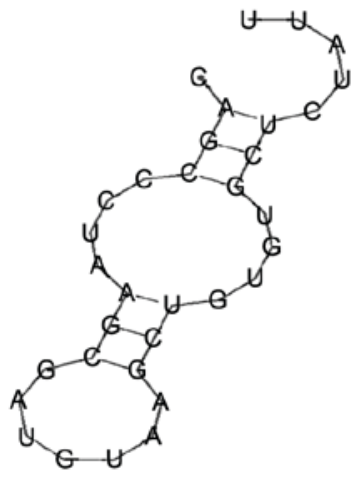

B

Sig pathway of target mRNAs

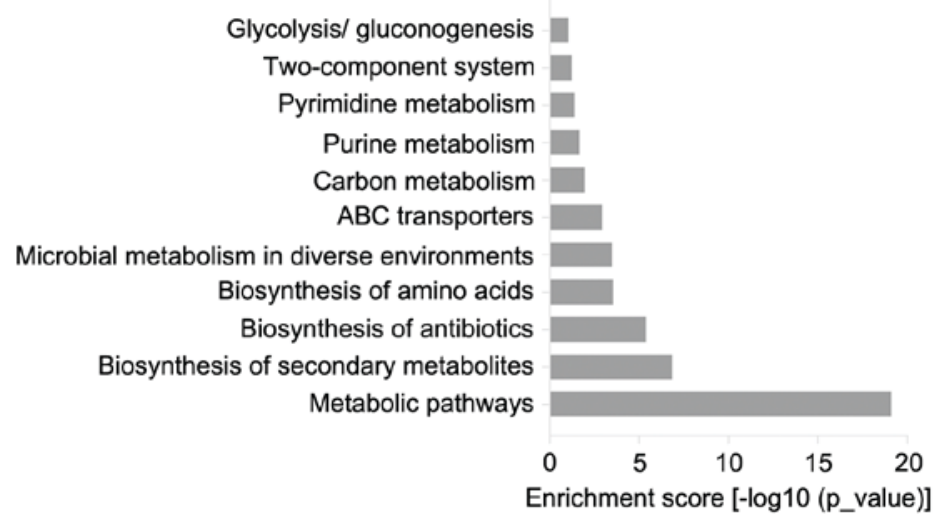

Figure 6. (A) Prediction of sRNA133474 lowest-energy secondary structure using RNAfold. sRNA133474 possessed a stem loop structure with the dG value of $-5.4 \mathrm{~kJ} / \mathrm{mol}$. (B) Biological pathways from Kyoto Encyclopedia of Genes and Genomes analysis with $\mathrm{P}<0.05$. sRNA, small noncoding RNA.

responses at the post-transcriptional level in several bacterial species (34). Thousands of sRNAs have been confirmed in S. mutans UA159; however, identification and function analyses of acid tolerance-associated sRNAs within clinical isolates are yet to be determined. In the present study, acid tolerance-associated sRNA133474 within clinical isolates of
S. mutans was identified and functional mechanisms were explored.

sRNA133474 expression patterns at specific growth phases in UA159 and clinical isolates were analyzed. These data revealed that the sequence of sRNA133474 was conserved among S. mutans strains and sRNA133474 
A

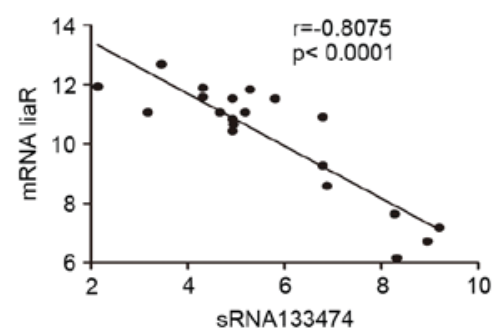

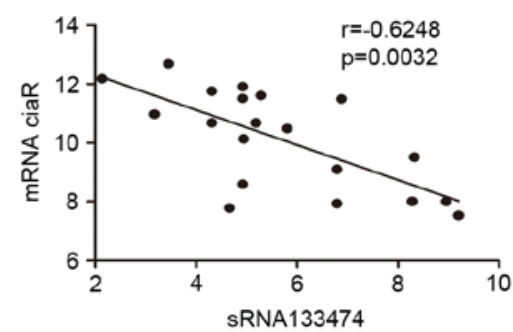

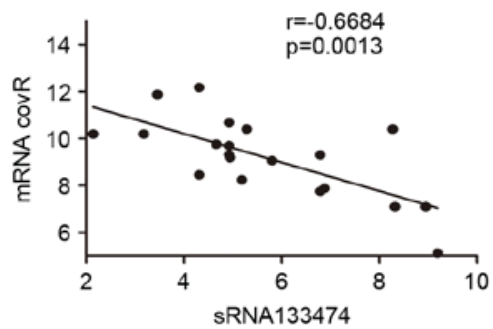

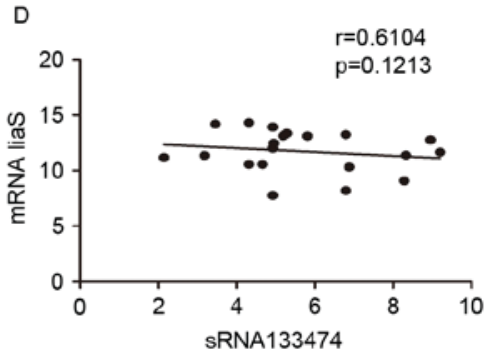

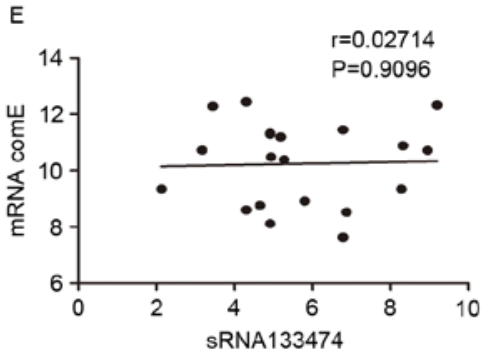

Figure 7. Spearman correlation analysis of the relationship between sRNA133474 and target mRNAs: (A) liaR, (B) $\operatorname{cia} R$, (C) $\operatorname{cov} R$, (D) liaS and (E) $\operatorname{comE}$ in clinical isolates. sRNA, small noncoding RNA.
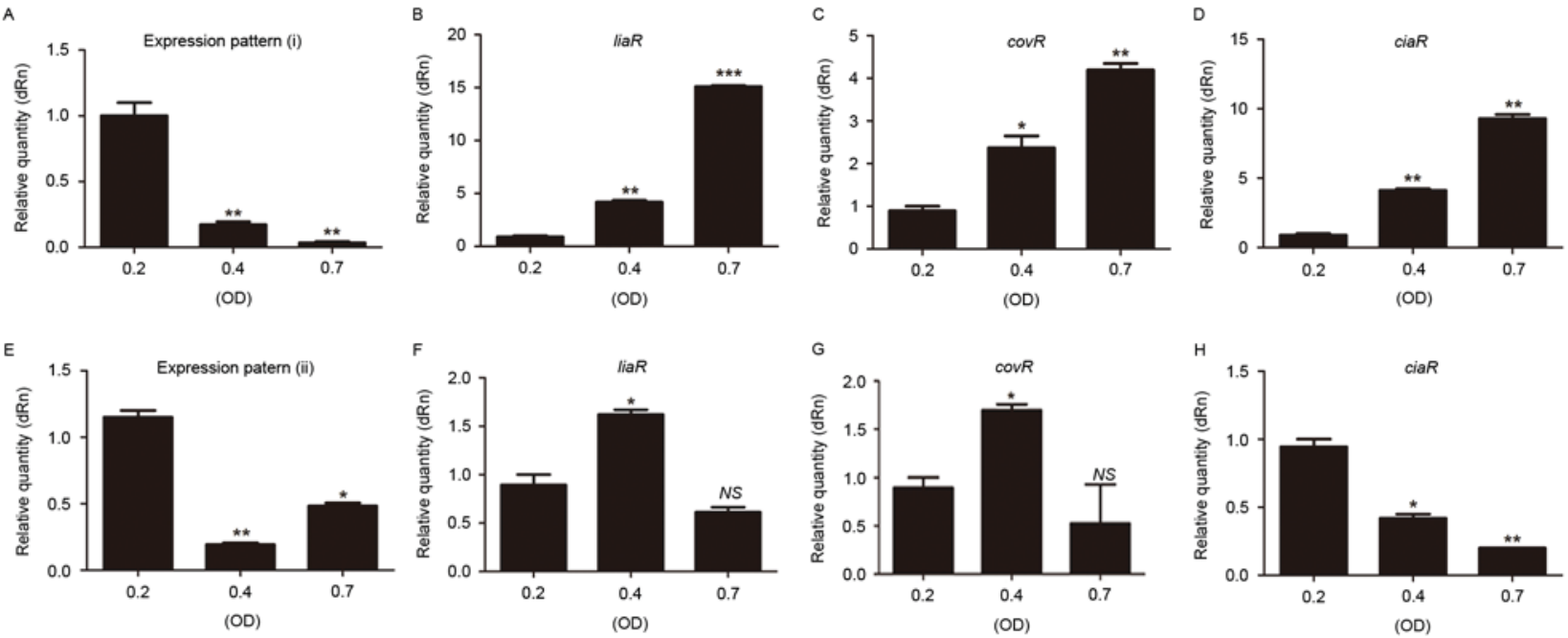

Figure 8. Expression of sRNA133474 and target mRNAs (liaR, covR and ciaR) in clinical strains with (A-D) expression pattern (i) and (E-H) expression pattern (ii). Data are presented as the mean \pm standard deviation. ${ }^{*} \mathrm{P}<0.05,{ }^{* *} \mathrm{P}<0.01,{ }^{* * * *} \mathrm{P}<0.001,{ }^{\mathrm{NS}} \mathrm{P}>0.05$ compared with the relative quantity at $\mathrm{OD}{ }_{600}=0.2$. (A, B and E) Data were analyzed using Kruskal-Wallis test and Bonferroni multiple comparisons test; (C, D, F, G and H) data were analyzed by analysis of variance and Bonferroni multiple comparisons test. NS, not significant; OD, optical density; sRNA, small noncoding RNA; dRn, delta Rn: The magnitude of the fluorescence signal generated during the PCR at each time point.

abundance was growth phase-dependent, consistent with previous studies (35-37). Several sRNAs have been reported to be expressed in a manner dependent on Staphylococcus aureus growth phases (37). Similarly, sRNA BTH_s1 and sRNA s39 expression levels were altered at different growth phases in Burkholderia thailandensis (35) and expression levels of three sRNAs in Listeria monocytogenes were also growth phase-dependent (36). Bacteria are homogenous during different growth stages and regulate factors to adapt their needs according to alterations in population density, cell cycles and environmental stressors, including $\mathrm{pH}$ fluctuations and nutrient availability $(38,39)$. S. mutans may employ sRNAs to regulate the responses to adverse environments and stress factors during different growth phases. Alterations in
sRNA133474 expression levels were consistent with decreasing $\mathrm{pH}$ during different growth phases. Therefore, acid tolerance of $S$. mutans may be associated with sRNA133474 expression.

The ability of $S$. mutans to respond to environmental stress conditions, such as acidic $\mathrm{pH}$, is essential for survival and cariogenicity. Expression levels of sRNA133474 in high- and low-acid tolerance groups were measured in the present study; sRNA133474 may serve a key role in regulating acid tolerance of S. mutans. The predicted target mRNAs of sRNA133474 comE, $\operatorname{cov} R$, liaS, liaR and ciaR have been reported to be imperative regulons of TCSs that contribute to acid tolerance in S. mutans.

Genes encoding CiaHR, LevSR, LiaSR, ScnKR, Hk/Rr1037/1038 and ComDE TCSs were upregulated during acid adaptation. The cia $\mathrm{R}$ gene encodes the cognate 
response regulator $\mathrm{CiaR}$ and contributes to acid tolerance. Strains of UA159 S. mutans with ciaR mutations exhibit a marked reduction in acid tolerance at pH 5.4 (40). The LiaSR (formerly HK/RR11) TCS in S. mutans has been reported to positively modulate acid tolerance. As previously reported, hkll mutants are growth defective at low $\mathrm{pH}$ and exhibit low acid tolerance; the acid tolerance-associated phenotype exhibited by $S$. mutans may be activated by $h k 11(14,34)$. In addition, $\operatorname{cov} R$ is deemed to be responsible for the virulence of $S$. mutans and contributes to acid tolerance (15). The negative correlation between sRNA133474 and target mRNAs (ciaR, liaR and $\operatorname{cov} R$ ) in clinical isolates suggested a negative regulatory role for sRNA133474 in $S$. mutans acid tolerance.

Studies of bacterial strains revealed that expression of regulatory genes is strain-specific $(23,39)$; the transcription patterns of response regulators (RR) vicR, $\operatorname{cov} R, \operatorname{com} E$, ciaR and $R R 1$ are reported to be strain-specific within clinical isolates of $S$. mutans (39). Differing expression profiles of target mRNA and sRNA133474 within clinical strains, as well as the observed negative association, were observed in the present study. These findings suggested that different transcriptional patterns of mRNA may result from heterogeneous expression patterns of sRNA133474. Altered patterns of sRNA133474 expression in clinical isolates may inhibit the expression of target mRNAs, contributing to distinct virulence properties (24). Isolates of $S$. aureus with different sRNAs and target expression levels exhibit varying pathogenesis and infective severity. Therefore, sRNA133474 may regulate mRNA covR/liaR/ciaR expression in LiaSR, CiaRH and CovRS TCS pathways responsible for acid tolerance of $S$. mutans. Consistent with the present study, Laux et al (41) reported that the expression of the response regulator ciaR was regulated by five similar sRNAs in S. pneumoniae R6.

In summary, this is the first study to explore the function and diversity of sRNA expression patterns in clinical isolates of $S$. mutans. The findings of the present study suggested that sRNA133474 and its target mRNAs are involved in TCSs and serve critical roles in the regulation of acid tolerance in clinical isolates of S. mutans. However, it is worthwhile to point out that the limitations in this study include the analysis of a limited number of sRNAs and clinical strains. Furthermore, the underlying mechanisms remain to be elucidated. The present study indicated that strain-specific expression patterns with sRNAs are associated with inter-strain variation and sRNA function.

Our previous study (25) revealed that other sRNAs are differentially expressed within acidic environments. Future investigations aim to determine the sRNAs involved in the acid response exhibited by $S$. mutans, and to understand the molecular mechanisms and regulation of these molecules. This research may be useful in the identification of potential biomarkers of dental caries to aid in prevention and targeted therapy.

\section{Acknowledgements}

The present study was supported by a grant from the National Natural Science Foundation of China (grant no. 81570967).

\section{References}

1. Selwitz RH, Ismail AI and Pitts NB: Dental caries. Lancet 369: 51-59, 2007.

2. Marsh PD: Microbial ecology of dental plaque and its significance in health and disease. Adv Dent Res 8: 263-271, 1994.

3. Dye BA and Thornton-Evans G: Trends in oral health by poverty status as measured by Healthy People 2010 objectives. Public Health Rep 125: 817-830, 2010.

4. Capurro DA, Iafolla T, Kingman A, Chattopadhyay A and Garcia I: Trends in income-related inequality in untreated caries among children in the United States: Findings from NHANES I, NHANES III and NHANES 1999-2004. Community Dent Oral Epidemiol 43: 500-510, 2015.

5. Qi X: Report of the third national oral health survey in China. People's Med Publ House, Beijing, pp60-61, 2008 (In Chinese).

6. Guo L, McLean JS, Lux R, He X and Shi W: The well-coordinated linkage between acidogenicity and aciduricity via insoluble glucans on the surface of Streptococcus mutans. Sci Rep 5: 18015, 2015.

7. Quivey RG, Kuhnert WL and Hahn K: Genetics of acid adaptation in oral streptococci. Crit Rev Oral Biol Med 12: 301-314, 2001.

8. Matsui R and Cvitkovitch D: Acid tolerance mechanisms utilized by Streptococcus mutans. Future Microbiol 5: 403-417, 2010.

9. Loesche WJ: Role of Streptococcus mutans in human dental decay. Microbiol Rev 50: 353-380, 1986.

10. Gross EL, Beall CJ, Kutsch SR, Firestone ND, Leys EJ and Griffen AL: Beyond Streptococcus mutans: Dental caries onset linked to multiple species by $16 \mathrm{~S}$ rRNA community analysis. PLoS One 7: e47722, 2012.

11. Lemos JA, Quivey RG Jr, Koo H and Abranches J: Streptococcus mutans: A new Gram-positive paradigm? Microbiology 159: 436-445, 2013.

12. Lévesque CM, Mair RW, Perry JA, Lau PC, Li YH and Cvitkovitch DG: Systemic inactivation and phenotypic characterization of two-component systems in expression of Streptococcus mutans virulence properties. Lett Appl Microbiol 45: 398-404, 2007.

13. Song L, Sudhakar P, Wang W, Conrads G, Brock A, Sun J, Wagner-Döbler I and Zeng AP: A genome-wide study of two-component signal transduction systems in eight newly sequenced mutans streptococci strains. BMC Genomics 13: 128, 2012.

14. Li YH, Lau PC, Tang N, Svensäter G, Ellen RP and Cvitkovitch DG: Novel two-component regulatory system involved in biofilm formation and acid resistance in Streptococcus mutans. J Bacteriol 184: 6333-6342, 2002.

15. Gong Y, Tian XL, Sutherland T, Sisson G, Mai J, Ling J and Li YH: Global transcriptional analysis of acid-inducible genes in Streptococcus mutans: Multiple two-component systems involved in acid adaptation. Microbiology 155: 3322-3332, 2009.

16. Senadheera D, Krastel K, Mair R, Persadmehr A, Abranches J, Burne RA and Cvitkovitch DG: Inactivation of VicK affects acid production and acid survival of Streptococcus mutans. J Bacteriol 191: 6415-6424, 2009.

17. Chong P, Drake L and Biswas I: LiaS regulates virulence factor expression in Streptococcus mutans. Infect Immun 76: 3093-3099, 2008.

18. Ortega AD, Quereda JJ, Pucciarelli MG and Garcia-del Portillo F: Non-coding RNA regulation in pathogenic bacteria located inside eukaryotic cells. Front Cell Infect Microbiol 4: $162,2014$.

19. Gottesman S and Storz G: Bacterial small RNA regulators: versatile roles and rapidly evolving variations. Cold Spring Harb Perspect Biol 3: pii: a003798, 2011.

20. Marx P, Nuhn M, Kovács M, Hakenbeck R and Brückner R: Identification of genes for small non-coding RNAs that belong to the regulon of the two-component regulatory system $\mathrm{CiaRH}$ in Streptococcus. BMC Genomics 11: 661, 2010.

21. Brosse A, Korobeinikova A, Gottesman S and Guillier M: Unexpected properties of sRNA promoters allow feedback control via regulation of a two-component system. Nucleic Acids Res 44: 9650-9666, 2016.

22. Saxena D, Li Y and Caufield PW: Identification of unique bacterial gene segments from Streptococcus mutans with potential relevance to dental caries by subtraction DNA hybridization. J Clin Microbiol 43: 3508-3511, 2005. 
23. Yu LX, Tao Y, Qiu RM, Zhou Y, Zhi QH and Lin HC: Genetic polymorphisms of the sortase A gene and social-behavioural factors associated with caries in children: A case-control study. BMC Oral Health 15: 54, 2015.

24. Stipp RN, Goncalves RB, Hofling JF, Smith DJ and Mattos-Graner RO: Transcriptional analysis of gtfB, gtfC, and $\mathrm{gbpB}$ and their putative response regulators in several isolates of Streptococcus mutans. Oral Microbiol Immunol 23: 466-473, 2008.

25. Liu S, Tao Y, Yu L, Zhuang P, Zhi Q, Zhou Y and Lin H: Analysis of Small RNAs in Streptococcus mutans under acid stress-A new insight for caries research. Int J Mol Sci 17: pii: E1529, 2016.

26. Cho SH, Lei R, Henninger TD and Contreras LM: Discovery of ethanol-responsive small RNAs in Zymomonas mobilis. Appl Environ Microbiol 80: 4189-4198, 2014.

27. Yin $\mathrm{H}, \mathrm{Hu} \mathrm{M}$, Zhang $\mathrm{R}$, Shen $\mathrm{Z}$, Flatow $\mathrm{L}$ and You $\mathrm{M}$ : MicroRNA-217 promotes ethanol-induced fat accumulation in hepatocytes by down-regulating SIRT1. J Biol Chem 287: 9817-9826, 2012

28. Yen YC, Shiah SG, Chu HC, Hsu YM, Hsiao JR, Chang JY, Hung WC, Liao CT, Cheng AJ, Lu YC and Chen YW: Reciprocal regulation of microRNA-99a and insulin-like growth factor I receptor signaling in oral squamous cell carcinoma cells. Mo Cancer 13: 6, 2014.

29. Ogata Y, Matsui S, Kato A, Zhou L, Nakayama Y and Takai H: MicroRNA expression in inflamed and noninflamed gingival tissues from Japanese patients. J Oral Sci 56: 253-260, 2014.

30. Livak KJ and Schmittgen TD: Analysis of relative gene expression data using real-time quantitative PCR and the 2(-Delta Delta C(T)) method. Methods 25: 402-408, 2001.

31. Eggenhofer F, Tafer H, Stadler PF and Hofacker IL: RNApredator: Fast accessibility-based prediction of sRNA targets. Nucleic Acids Res 39: W149-W154, 2011.

32. Xia L, Xia W, Li S, Li W, Liu J, Ding H, Li J, Li H, Chen Y, Su X, et al: Identification and expression of small non-coding RNA, L10-Leader, in different growth phases of Streptococcus mutans. Nucleic Acid Ther 22: 177-186, 2012.
33. Lian C, Sun B, Niu S, Yang R, Liu B, Lu C, Meng J, Qiu Z, Zhang $\mathrm{L}$ and Zhao $\mathrm{Z}$ : A comparative profile of the microRNA transcriptome in immature and mature porcine testes using Solexa deep sequencing. FEBS J 279: 964-975, 2012.

34. Suntharalingam P, Senadheera MD, Mair RW, Lévesque CM and Cvitkovitch DG: The LiaFSR system regulates the cell envelope stress response in Streptococcus mutans. J Bacteriol 191: 2973-2984, 2009.

35. Stubben CJ, Micheva-Viteva SN, Shou Y, Buddenborg SK Dunbar JM and Hong-Geller E: Differential expression of small RNAs from Burkholderia thailandensis in response to varying environmental and stress conditions. BMC Genomics 15: 385 , 2014.

36. Christiansen JK, Larsen MH, Ingmer H, Søgaard-Andersen L and Kallipolitis BH: The RNA-binding protein Hfq of Listeria monocytogenes: Role in stress tolerance and virulence. J Bacteriol 186: 3355-3362, 2004.

37. Bronsard J, Pascreau G, Sassi M, Mauro T, Augagneur Y and Felden B: sRNA and cis-antisense sRNA identification in Staphylococcus aureus highlights an unusual sRNA gene cluster with one encoding a secreted peptide. Sci Rep 7: 4565, 2017.

38. Ahn SJ, Lemos JA and Burne RA: Role of HtrA in growth and competence of Streptococcus mutans UA159. J Bacteriol 187: 3028-3038, 2005.

39. Tremblay YD, Lo H, Li YH, Halperin SA and Lee SF: Expression of the Streptococcus mutans essential two-component regulatory system VicRK is $\mathrm{pH}$ and growth-phase dependent and controlled by the LiaFSR three-component regulatory system. Microbiology 155: 2856-2865, 2009.

40. Ahn SJ, Wen ZT and Burne RA: Multilevel control of competence development and stress tolerance in Streptococcus mutans UA159. Infect Immun 74: 1631-1642, 2006.

41. Laux A, Sexauer A, Sivaselvarajah D, Kaysen A and Brückner R: Control of competence by related non-coding csRNAs in Streptococcus pneumoniae R6. Front Genet 6: 246, 2015. 